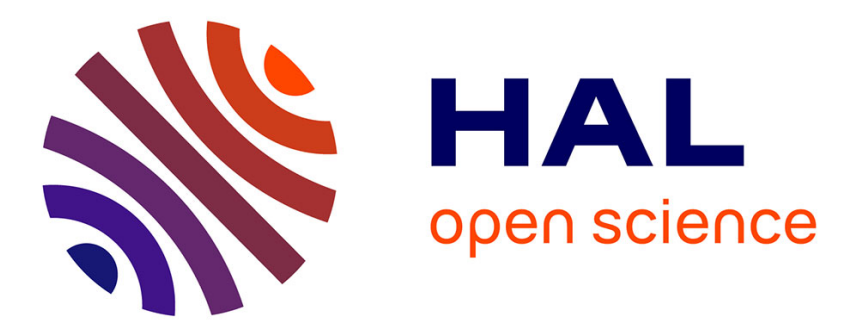

\title{
Embeddings into the Pancake Interconnection Network
}

Christian Lavault

\section{To cite this version:}

Christian Lavault. Embeddings into the Pancake Interconnection Network. Parallel Processing Letters, 2002, 12 (3-4), pp.297-310. hal-00003362

\section{HAL Id: hal-00003362 \\ https://hal.science/hal-00003362}

Submitted on 26 Nov 2004

HAL is a multi-disciplinary open access archive for the deposit and dissemination of scientific research documents, whether they are published or not. The documents may come from teaching and research institutions in France or abroad, or from public or private research centers.
L'archive ouverte pluridisciplinaire HAL, est destinée au dépôt et à la diffusion de documents scientifiques de niveau recherche, publiés ou non, émanant des établissements d'enseignement et de recherche français ou étrangers, des laboratoires publics ou privés. 


\title{
Embeddings into the Pancake Interconnection Network
}

\author{
Christian LAVAULT \\ LIPN, CNRS ESA 7030, Université Paris 13 \\ 99, av. J.-B. Clément 93430 Villetaneuse, France \\ E-mail: lavault@lipn.univ-paris13.fr
}

\begin{abstract}
Owing to its nice properties, the pancake is one of the Cayley graphs that were proposed as alternatives to the hypercube for interconnecting processors in parallel computers. In this paper, we present embeddings of rings, grids and hypercubes into the pancake with constant dilation and congestion. We also extend the results to similar efficient embeddings into the star graph.
\end{abstract}

\section{Introduction}

Akers and Krishnamurthy [1] proposed the pancake and the star as alternatives to the hypercube for interconnecting processors in parallel computers. These networks have some nice properties: edge and vertex symmetry (strong symmetry), small degree and diameter, extensibility, high connectivity (robustness), easy routings and broadcasting, etc. To compare favorably with the hypercube, these graphs must also offer good and simple simulations of other interconnection networks. The problem of simulating known networks by the star graph has been extensively studied. For example, Nigam, Sahni and Krishnamurthy [8] consider embeddings of rings and hypercubes in star graphs. Miller, Pritkin, and Sudborough [7] study one-to-one and one-to-many embeddings of hypercubes into Cayley graphs. Jwo, Lakshmivarahan, and Dhall [6], Qiu, Meijer, and Akl [9, 10] consider embeddings of grids in stars. Bouabdallah, Heydemann, Opatrny and Sotteau [5] present embeddings of complete binary tree into star networks. Azevedo, Bagherzadeh and Latifi [0] propose embeddings of hypercubes in star graphs. 
However, embedding interconnection networks into the pancake has received less attention. In this paper, we focus on the problem and present embeddings of rings, grids and hypercubes into the pancake with constant dilation and congestion.

The paper is organized as follows. In the Preliminaries, we state the definitions and the group-theoretic terminology that are used in this paper. Section 3 is devoted to embeddings of rings and arrays into the pancake. In Section 4, we present embeddings of grids into the pancake and the star, and in Section 5, we consider embeddings of hypercubes (binary and generalized hypercubes) into the pancake; the results are extended to the star. The concluding Section 6 briefly outlines possible improvements and open problems.

\section{Preliminaries}

Following [1, 4, we first present the group-theoretic model used to design and analyze the pancake. Next we define the pancake network itself.

Definition 1 Let $\mathcal{G}$ be a finite multiplicative group. Let $I$ be the identity in $\mathcal{G}$ and $G$ a set of generators of $\mathcal{G}$ with the following two properties

(i) $(\forall g \in G) \quad g^{-1} \in G$;

(ii) $I \notin G$.

Given $(\mathcal{G}, G)$, a Cayley graph $(V, E)$ is defined as a simple graph, whose vertex-set and edge-set are

$$
V=\mathcal{G} \quad \text { and } \quad E=\left\{(u, v) \in V \times V \mid u^{-1} v \in G\right\} .
$$

It is easily seen that Cayley graphs $(V, E)$ are finite, connected, undirected, devoid of multiple edges, loop-free, and symmetric. Since interconnection networks may be viewed as an undirected graph, we will use the terms graph and (interconnection) network interchangeably.

Notation. In the remainder of the paper, we use the usual terminology of basic group theory and graph theory. Since we only consider finite groups, the groups are mainly represented as permutation groups. The following notation is used:

- Let $X$ and $Y$ be two sets, $X \backslash Y$ denotes the relative complement of the set $Y$ with respect to the set $X$.

- $S_{n}$ is the symmetric group on $n$ symbols, i.e. on $\{1, \ldots, n\}$ for simplicity. The multiplication in $S_{n}$ is the composition of permutations. 
A permutation $\pi \in S_{n}$ is denoted by $x_{1} x_{2} \cdots x_{n}$, where we have $\pi(k)=x_{k}$ for $k=1, \ldots, n$. This representation is referred to as the standard representation of a permutation, to distinguish it from other representations that are introduced further. The identity permutation on $n$ symbols is thus $I=123 \cdots n$.

- $\tau$ denotes the transposition permutation and $\sigma(\ell, m)$ denotes the cyclic permutation of $m$ positions over the first $\ell$ symbols:

$$
\sigma(\ell, m)=(\ell-m+1) \cdots \ell 1 \cdots(\ell-m)(\ell+1) \cdots n .
$$

- Symbols are denoted by lower case letters, and blocks of symbols by upper case letters. No ambiguity may rise from the notation $I$, since $I$ is (by definition) the unique block of symbols $123 \cdots n$.

- A permutation $\pi=x_{1} x_{2} \cdots x_{i-1} x_{i} x_{i+1} \cdots x_{n}$ can be represented by blocks of symbols instead of its symbols themselves. For example, we can write $\pi=A B$, where $A=x_{1} x_{2} \cdots x_{i-1} x_{i}$ and $B=x_{i+1} \cdots x_{n}$.

For any block of symbols $A, \bar{A}$ denotes the block obtained by reversing $A$. For example, if $\rho=x_{i} x_{i-1} \cdots x_{2} x_{1} x_{i+1} \cdots x_{n}$, we write $\rho=\bar{A} B$, where $A$ and $B$ are the blocks defined above.

\section{Definition 2 [1]}

The pancake network $P_{n}$ of dimension $n$ is the Cayley graph $\left(S_{n}, E\right)$, whose set of generators is

$$
G=\left\{g_{i} \in S_{n} \mid g_{i}=i(i-1) \cdots 321(i+1) \cdots n, i=2, \ldots, n\right\} .
$$

In other words, the $n$ ! vertices of $P_{n}$ are labeled with the permutations on $n$ symbols (of $S_{n}$ ), and any two vertices of $P_{n}, u=x_{1} x_{2} \cdots x_{n}$ and $v=y_{1} y_{2} \cdots y_{n}$, are connected (i.e. $(u, v) \in E$ ) iff there exists an integer $i, 2 \leq i \leq n$, such that $y_{j}=x_{i-j+1}$ for $j=1, \ldots, i$, and $y_{j}=x_{j}$ for $j>i$.

Clearly, there are $(n-1)$ generators, one for each value of $i, 2 \leq i \leq n$, and $|G|=n-1$. It is easy to show that the Cayley network $P_{n}$ has $n$ ! vertices, each with degree $|G|=n-1: P_{n}$ is $(n-1)$-regular.

When a permutation $\rho$ is obtained from a permutation $\pi=x_{1} \cdots x_{n}$ by applying a generator $g_{i} \in G$, we write $x_{1} \cdots x_{i} \cdots x_{n} \rightarrow \underline{x_{i} \cdots x_{1}} \cdots x_{n}$.

$P_{n}$ can be decomposed into $n$ subpancakes each of dimension $(n-1)$. Each of the $(n-1)$ ! vertices of each subpancake has a block representation of the form $A i$, where $A \in S_{n-1}$ is a "permutations block" on the $(n-1)$ symbols $\{1, \ldots, n\} \backslash\{i\}$, for a given 
$i \in\{1, \ldots, n\}$ which depends on the considered subpancake. As a consequence, each of the $n$ subpancakes of $P_{n}$ (one for each value of $i, 1 \leq i \leq n$ ) can be represented with two distinct notations depending on the context:

1. For a given integer $i(1 \leq i \leq n), P_{n-1, i}$ denotes the subpancake defined from the above representation, i.e. $i$ is the last symbol of each vertex/permutation of $P_{n-1, i}$.

2. For a given permutation $\pi \in P_{n-1, i}(1 \leq i \leq n), P_{n-1}(\pi)$ denotes the subpancake defined from the element $\pi$, i.e. $\pi$ is the representation of a vertex of that subpancake.

A subpancake of dimension $k$ is called a $k$-pancake.

Definition 3 The star network of dimension n (or n-star) is the Cayley graph whose set of generators is $G=\{\tau(1, i) \mid i=1, \ldots, n\}$, where $\tau \in S_{n}$ is the transposition permutation. In other words, the $n$ ! vertices of the $n$-star are labeled with the permutations on $n$ symbols, and each vertex $u$ of the $n$-star, $u=x_{1} x_{2} \cdots x_{n}$ is connected to the $n-1$ vertices $v$ such that $v=x_{i} x_{2} \cdots x_{i-1} x_{1} x_{i+1} \cdots x_{n}$, for $i=2, \ldots, n$.

Now recall the definition of an embedding of a graph into another graph.

Definition 4 Given two undirected connected graphs $H_{1}=\left(V_{1}, E_{1}\right)$ and $H_{2}=$ $\left(V_{2}, E_{2}\right)$ such that $\left|V_{1}\right| \leq\left|V_{2}\right|$, the embedding of $H_{1}$ into $H_{2}$ maps $V_{1}$ into $V_{2}$. The ratio $\left|V_{2}\right| /\left|V_{1}\right|$ is the expansion of the embedding. The dilation of any edge $\left(x_{1}, y_{1}\right) \in E_{1}$, is the length of the path $\left[x_{2}, y_{2}\right]$, where $x_{1} \mapsto x_{2}$ and $y_{1} \mapsto y_{2}$ in the embedding, respectively. The dilation of the embedding is the maximum over all dilations. The congestion of an edge $\left(x_{2}, y_{2}\right) \in E_{2}$ is the number of edges $\left(x_{1}, y_{1}\right) \in E_{1}$ whose image by the mapping contains $\left(x_{2}, y_{2}\right)$. The congestion of the embedding is the maximum over all congestions.

\section{Embeddings of Rings}

In this section we consider rings of size $k !(3 \leq k \leq n)$. The dilation and the congestion of embeddings of such rings into $P_{n}$ are shown to be 1 .

Proposition 1 For any integer $i, 2 \leq i \leq n-1$, and any generator $g_{i} \in G$ as defined in Definition $8, \underbrace{g_{i} g_{i+1} \cdots g_{i} g_{i+1}}_{i+1}=I$. 
Proof. Let a permutation $\pi=x_{1} x_{2} \cdots x_{i-1} x_{i} x_{i+1} \cdots x_{n}=A x_{i+1} B$. Then $\rho=\pi g_{i}=\bar{A} x_{i+1} B$, and

$$
\pi g_{i} g_{i+1}=\rho g_{i+1}=x_{i+1} A B=x_{i+1} x_{1} x_{2} \cdots x_{i-1} x_{i} x_{i+2} \cdots x_{n}
$$

In other words, $g_{i} g_{i+1}$ is the cyclic permutation $\sigma(i+1,1)$, and since $\sigma(i+1,1)^{i+1}=I$, the result follows.

For any permutation $\pi$ and any sequence of generators $H=h_{1}, \ldots, h_{k}$, we denote by $(\pi, H)$ the corresponding sequence of permutations $\pi_{0}, \ldots, \pi_{k}$ such that $\pi_{0}=\pi$ and $\pi_{i}=\pi_{i-1} h_{i}$, for all $i=1,2, \ldots, k$.

Definition 5 For $k=2, \ldots, n$, the pancake sequence $G_{k}$ of order $k$ is the sequence of generators recursively defined as follows:

(i) $G_{2}=g_{2}$;

(ii) for $k>2, G_{k}=\left\langle G_{k-1}, g_{k}, G_{k-1}, g_{k}, \ldots, G_{k-1}\right\rangle$, where $G_{k-1}$ occurs $k$ times in the sequence.

Proposition 2 Given a permutation $\pi \in S_{n}$, for $k=3, \ldots, n,\left(\pi, G_{k}\right)$ defines a Hamiltonian cycle over the k-pancake containing $\pi$. In this Hamiltonian cycle, the vertices of each subpancake have adjacent locations.

Proof. The proof is by induction on $k$. Since the pancake is vertex transitive, we assume that $\pi=I$.

- Base $(k=3)$ : Applying the generators of $G_{3}$ yields the following sequence of permutations:

$$
I=123 \cdots n \rightarrow \underline{213} \cdots n \rightarrow \underline{312} \cdots n \rightarrow \underline{132} \cdots n \rightarrow \underline{231} \cdots n \rightarrow \underline{321} \cdots n .
$$

It is easily verified that all the elements of $P_{3}(I)$ belong to the sequence and that the last element of the list is connected to the first one through the generator $g_{3}$.

- Induction step: Suppose that $\left(\pi, G_{n-1}\right)$ defines a Hamiltonian cycle over $P_{n-1}(\pi)$. We first show that the permutation obtained by applying $\left\langle G_{n-1}, g_{n}, \ldots, G_{n-1}, g_{n}\right\rangle$ ( $h$ times) is $(n-h+1)(n-h+2) \cdots n 12 \cdots(n-h)$. The property holds for $h=1$ since the permutation is obtained from the sequence

$$
12 \cdots(n-1) n \rightarrow \underline{(n-1) \cdots 21} n \rightarrow \underline{n 12 \cdots(n-1)} .
$$

Let us now suppose that the property holds up to $h$. The next step is then

$$
\begin{aligned}
& (n-h+1)(n-h+2) \cdots n 12 \cdots(n-h-1)(n-h) \rightarrow \\
& \underline{(n-h-1) \cdots 21 n \cdots(n-h+1)}(n-h) \rightarrow \underline{(n-h) \cdots n 12 \cdots(n-h-1)} .
\end{aligned}
$$


Therefore, after applying $\left\langle G_{n-1}, g_{n}, \ldots, G_{n-1}, g_{n}\right\rangle$ ( $h$ times), the permutation corresponds to a vertex of $P_{n-1}(n-h)$. According to the induction step, the next $G_{n-1}$ visits all the vertices of the subpancake $P_{n-1}(n-h)$. Whence the result that all vertices of all the $(n-1)$-pancakes in $P_{n}$ are visited. The last visited vertex is $(2 \cdots n 1) g_{n-1}=n \cdots 21$, and this permutation is connected to $I$ through $g_{n}$ : the proof is completed.

In the following, we still let $\pi=I$ (w.l.o.g.). The order relation induced by the sequence $\left(I, G_{n}\right)$ on permutations will be referred to as the ordering of the pancake sequence.

Example. Let $n=4$. The list of vertices of $P_{4}$ ordered by the pancake sequence is: $1234 \rightarrow \underline{21} 34 \rightarrow \underline{312} 4 \rightarrow \underline{13} 24 \rightarrow \underline{2314} \rightarrow \underline{3214} \rightarrow \underline{4123} \rightarrow \underline{14} 23 \rightarrow \underline{2413} \rightarrow \underline{42} 13 \rightarrow$ $\underline{124} 3 \rightarrow \underline{21} 43 \rightarrow \underline{3412} \rightarrow \underline{43} 12 \rightarrow \underline{1342} \rightarrow \underline{3142} \rightarrow \underline{4132} \rightarrow \underline{14} 32 \rightarrow \underline{2341} \rightarrow \underline{32} 41 \rightarrow$ $\underline{4231} \rightarrow \underline{24} 31 \rightarrow \underline{3421} \rightarrow \underline{43} 21$.

Theorem 1 easily follows.

Theorem 1 For $k=3, \ldots, n$, the ring of size $k$ ! can be embedded into the $n$-pancake with dilation 1 and congestion 1.

Proof. Immediate from Proposition 2. Given a Hamiltonian graph of order $n$, the corresponding ring can be embedded into that graph with dilation and congestion 1 .

As a consequence of Theorem 1, we also have the

Corollary 1 For $\ell$ such that $\ell \leq n$ !, the linear array (line) of length $\ell$ can be embedded into the $n$-pancake with dilation 1 and congestion 1.

\section{Embeddings of Grids}

\subsection{Embeddings of $N_{1} \times N_{2}$ Grids}

Given any two positive integers $N_{1}$ and $N_{2}$, we first consider embeddings of $N_{1} \times N_{2}$ grids with $N_{1} N_{2} \leq n$ ! into $P_{n}$ and give a negative result.

Proposition 3 The $N_{1} \times N_{2}$ grid is not a subgraph of the n-pancake. 
Proof. The proof is by contradiction. The $2 \times 2$ grid is a subgraph of the $N_{1} \times N_{2}$ grid. Suppose the $2 \times 2$ grid were a subgraph of $P_{n}$, then there would be two permutations $X$ and $Y$, and four generators $g_{i}, g_{j}, g_{\ell}, g_{k}$, with $i \neq j, i \neq k$, $k \neq \ell$, such that $Y=X g_{i}$ and $Y g_{k}=X g_{j} g_{\ell}$. Hence, $g_{i} g_{k}=g_{j} g_{\ell}$, which would imply that $j=\ell$ and $k=i$, or $j=i$ and $\ell=k$ : a contradiction.

Lemma 1 For any two integers $\ell$ and $m$ such that $0 \leq m \leq \ell \leq n$, the cyclic permutation $\sigma(\ell, m)$ can always be built with two or three generators of the pancake.

Proof. Let a permutation $\pi=x_{1} \cdots x_{\ell-m} x_{\ell-m+1} \cdots x_{\ell} x_{\ell+1} \cdots x_{n}=A B C$, with blocks $A=x_{1} \cdots x_{\ell-m}, B=x_{\ell-m+1} \cdots x_{\ell}$ and $C=x_{\ell+1} \cdots x_{n}$. Then, $\pi \sigma(\ell, m)=x_{\ell-m+1} \cdots x_{\ell} x_{1} \cdots x_{\ell-m} x_{\ell+1} \cdots x_{n}=B A C$, and we have the following path joining $\pi$ to $\pi \sigma(\ell, m): \pi=A B C \rightarrow \bar{A} B C \rightarrow \bar{B} A C \rightarrow B A C=\pi \sigma(\ell, m)$. The length of this path is 3 when $1<m<\ell-1$, and it is 2 whenever $m=1$ or $m=\ell-1$.

Now from Lemma 11 we present an embedding of the $n \times(n-1)$ ! grid in $P_{n}$ with constant dilation.

Theorem 2 The $n \times(n-1)$ ! grid can be embedded in the $n$-pancake with dilation 7 .

Proof. The first row of the grid is represented by the first $(n-1)$-pancake ordered from the pancake sequence. For $0 \leq j \leq(n-1)$ ! -1 , let $\pi_{j}$ be the vertex of the pancake corresponding to the node $(0, j)$ on the grid. A node $(i, j)$, with $i \neq 0$, is represented by $\pi_{j} \sigma(n, i)$. Now, considering two adjacent nodes on the grid, let us compute the distance between those vertices of the pancake that represent them.

- Two nodes $(0, j)$ and $(0, j+1)$ are represented by two adjacent vertices of the pancake.

- Two nodes $(i, j)$ and $(i+1, j)$ are represented by the two vertices $X=\pi_{j} \sigma(n, i)$ and $Y=\pi_{j} \sigma(n, i+1)=X \sigma(n, 1)$. According to Lemma 1, the distance between $X$ and $Y$ is 2 .

- Two nodes $(i, j)$ and $(i, j+1)$ are represented by the two vertices $Y_{1}=\pi_{j} \sigma(n, i)$ and $Y_{2}=\pi_{j+1} \sigma(n, i)$. Then, for a generator $g_{k}, \pi_{j+1}=\pi_{j} g_{k}$. To compute the distance between $Y_{1}$ and $Y_{2}$, two distinct cases (and two subcases) may arise:

- First case: $\pi_{j}=A B C, \pi_{j+1}=\bar{A} B C$ and $Y_{1}=C A B$. Then, $Y_{2}=C \bar{A} B$, and a path joining $Y_{1}$ to $Y_{2}$ is $C A B \rightarrow \bar{A} \bar{C} B \rightarrow A \bar{C} B \rightarrow C \bar{A} B$. The distance from $Y_{1}$ to $Y_{2}$ is 3 . 
- Second case: $\pi_{j}=A B C, \pi_{j+1}=\bar{B} \bar{A} C$, and $Y_{1}=B C A$. To obtain $Y_{2}$, two subcases must be considered.

- First subcase: $Y_{2}=\bar{B}_{1} \bar{A} C \bar{B}_{2}$. In this subcase, a path from $Y_{1}$ to $Y_{2}$ is $Y_{1}=B_{1} B_{2} C A \rightarrow \bar{B}_{2} \bar{B}_{1} C A \rightarrow B_{2} \bar{B}_{1} C A \rightarrow \bar{A} \bar{C} B_{1} \bar{B}_{2} \rightarrow C A B_{1} \bar{B}_{2} \rightarrow$ $\bar{C} A B_{1} \bar{B}_{2} \rightarrow \bar{B}_{1} \bar{A} C \bar{B}_{2}=Y_{2}$. The distance from $Y_{1}$ to $Y_{2}$ is 6 .

- Second subcase: $Y_{2}=\bar{A}_{1} C \bar{B} \bar{A}_{2}$. In this last subcase a path from $Y_{1}$ to $Y_{2}$ is $Y_{1}=B C A_{1} A_{2} \rightarrow \bar{A}_{2} \overline{A_{1}} \bar{C} \bar{B} \rightarrow A_{2} \overline{A_{1}} \bar{C} \bar{B} \rightarrow B C A_{1} \bar{A}_{2} \rightarrow \bar{C} \bar{B} A_{1} \bar{A}_{2}$ $\rightarrow C \bar{B} A_{1} \bar{A}_{2} \rightarrow B \bar{C} A_{1} \bar{A}_{2} \rightarrow \bar{A}_{1} C \bar{B} \bar{A}_{2}=Y_{2}$.

This last configuration yields a (maximal) distance 7 from $Y_{1}$ to $Y_{2}$.

The same method applies to the $(n+(n-2)+(n-3)+\cdots+(p+1)) \times p$ ! grid, for $p=2, \ldots, n-1$; the following Theorem 0 shows that this grid can be embedded into $P_{n}$ with constant dilation. Note that the term $(n-1)$ is omitted in the definition of the grid. Indeed, by Proposition 3, we already know that the $(n+(n-1)+(n-2)+\cdots+(p+1)) \times p ! \operatorname{grid}(2 \leq p \leq n-1)$ is not a subgraph of $P_{n}$.

Theorem 3 For $p=2, \ldots, n-1$, the $(n+(n-2)+(n-3)+\cdots+(p+1)) \times p$ ! grid can be embedded in the $n$-pancake with dilation 4.

Proof. The first row of the mesh is represented by the first $p$-pancake. The next $(n-1)$ rows are obtained by applying the cyclic permutations $\sigma(n, i)$ to the first row. The next $(n-2)$ rows are obtained by applying the cyclic permutations $\sigma(n-1, i)$ to the first row, etc. Finally, the last $p$ rows are obtained by applying the cyclic permutations $\sigma(p+1, i)$ to the first row. The only new adjacent nodes to consider are $X \sigma(k, k-1)$ and $X \sigma(k-1,1)$. Let $X=x_{1} A x_{k-1} x_{k} B$. Then, $X \sigma(k, k-1)=A x_{k-1} x_{k} x_{1} B$ and $X \sigma(k-1,1)=x_{k-1} x_{1} A x_{k} B$. A path joining these two vertices is

$$
\begin{aligned}
& A x_{k-1} x_{k} x_{1} B \rightarrow x_{k} x_{k-1} \bar{A} x_{1} B \rightarrow x_{1} A x_{k-1} x_{k} B \\
& \quad \rightarrow \bar{A} x_{1} x_{k-1} x_{k} B \rightarrow x_{k-1} x_{1} A x_{k} B .
\end{aligned}
$$

The distance between the two vertices is thus 4 and the proof follows.

\subsection{Embeddings of $n$-Grids}

For embedding $n$-grids into $P_{n}$, a new representation of permutations is first introduced. A permutation $\pi$ may be represented as $\pi=a_{2} a_{3} \cdots a_{n}$, where $a_{i}$ is the number of symbols less than $i$ that are located at the left of $i$ in the standard representation of $\pi$. 
Example. Let $n=5$. The permutation 12345 is represented by 1234 , and the permutation 54321 is represented by 0000. Similarly, the permutation 42153 is represented by 0203 .

The map $S_{n} \longrightarrow\left\{\pi=a_{2}, \ldots, a_{n} \mid 0 \leq a_{i} \leq i-1\right\}$ is obviously one-one, and it is used in this subsection to embed the $2 \times 3 \times \cdots \times(n-1) \times n$ grid into $P_{n}$.

Theorem 4 The $2 \times 3 \times \cdots \times(n-1) \times n$ grid can be embedded into the $n$-pancake with dilation 6.

Proof. Let two vertices $X$ and $Y$ on the grid be labeled with the two permutations $a_{2} \cdots a_{i-1} \alpha a_{i+1} \cdots a_{n}$ and $a_{2} \cdots a_{i-1} \beta a_{i+1} \cdots a_{n}$, respectively. $X$ and $Y$ are connected on the grid iff $\alpha=\beta+1$ or $\alpha=\beta-1$. Let us find the distance from $X$ to $Y$ in the pancake.

We may assume w.l.o.g. that $\alpha=\beta+1$. Let $X=A x_{k} B i C$, where $x_{k}$ is the $\alpha$ th symbol $<i$ and all symbols in $B$ are $>i$. Consider the permutation $A i B x_{k} C=$ $b_{2} \cdots b_{n}$ and compare the $a_{j}$ s and the $b_{j}$ s.

First, for all symbols in $A$ and $C, b_{j}=a_{j}$. Next, for each symbol $j$ in $B$, a new symbol that is smaller than $j$ is located on the left of $j$ : it is the symbol $i$. Similarly, a new symbol that is smaller than $j$ is located on the right of $j$ : it is the symbol $x_{k}$. Hence, $b_{j}=a_{j}+1-1=a_{j}$. New symbols located on the left of $x_{k}$ are larger than $x_{k}$; they are either the symbol $i$ or any symbol $j>i$, and hence, $b_{x_{k}}=a_{x_{k}}$. There is only one new symbol smaller than $i$ located on the right of $i$ : it is the symbol $x_{k}$, other symbols are larger than $i$. Hence, $b_{i}=a_{i}-1=\alpha-1=\beta$.

Therefore, $A i B x_{k} C=Y$, and a path joining $X$ to $Y$ is

$$
\begin{aligned}
X & =A x_{k} B i C \rightarrow x_{k} \bar{A} B i C \rightarrow i \bar{B} A x_{k} C \rightarrow B i A x_{k} C \\
& \rightarrow \bar{B} i A x_{k} C \rightarrow \bar{A} i B x_{k} C \rightarrow A i B x_{k} C=Y .
\end{aligned}
$$

The distance from $X$ to $Y$ is thus 6 , and the dilation follows.

The following corollary is easily derived.

Corollary 2 The binary hypercube $Q_{n}$ can be embedded in the n-pancake with dilation 6.

Proof. $\quad Q_{n}$ is a subgraph of the $2 \times 3 \times \cdots \times(n-1) \times n$ grid.

The same method applies to embed the $n$-grid into the star graph (see Definition (3). 
Theorem 5 The $2 \times 3 \times \cdots \times(n-1) \times n$ grid can be embedded into the $n$-star with dilation 3.

Proof. The above representation of permutations is used again. Consider two permutations $X=a A x B y C$ and $Y=a A y B x C$, and compute the distance from $X$ to $Y$ within the $n$-star. A path joining $X$ to $Y$ is

$$
X=a A x B y C \rightarrow y A x B a C \rightarrow x A y B a C \rightarrow a A y B x C=Y .
$$

The distance from $X$ to $Y$ is thus 3, and the dilation follows.

\section{Embeddings of the Generalized Hypercube}

The $2 \times 3 \times \cdots \times(n-1) \times n$ generalized hypercube $(G H C)$ [3] is the graph $(V, E)$ whose vertices are labeled withthe permutations $x_{2}, \ldots, x_{n}$, where $0 \leq x_{i} \leq i-1$. Any two vertices $u, v \in V$ are connected iff their labels differ in only one position: i.e. there is an edge $(u, v) \in E$ between the two vertices $u=x_{2}, \ldots, x_{i-1}, \alpha, x_{i+1}, \ldots, x_{n}$ and $v=x_{2}, \ldots, x_{i-1}, \beta, x_{i+1}, \ldots, x_{n}$ iff $\alpha \neq \beta$ for some symbols $\alpha$ and $\beta$ from $x_{2}$ onwards.

Embedding the GHC into the $n$-pancake could be performed via the representation of permutations defined in the previous section. Unfortunately, the resulting dilation is $O(n)$, i.e. the dilation would then have the same order of magnitude as the diameter of the pancake. Consequently, a more suited representation of permutations must be used.

A permutation $\pi=x_{1} \cdots x_{n}$ is represented by $a_{2} \cdots a_{n}$ with the following rule $(\mathbf{R})$ :

for $k=n$ to 2 (step -1$)$ do

$a_{k} \leftarrow x_{k}-1 ; x_{k} \leftrightarrow k$ (i.e. exchange symbols $x_{k}$ and $k$ in permutation $\pi$ ).

Example. Let $n=8$ and $X=27351864$.Applying rule $(\mathbf{R})$ step by step yields

$a_{8}=4-1=3, \quad$ and $Y_{1}=27351468$,

$a_{7}=6-1=5, \quad$ and $Y_{2}=26351478$,

$a_{6}=4-1=3, \quad$ and $Y_{3}=24351678$,

$a_{5}=1-1=0, \quad$ and $Y_{4}=24315678$,

$a_{4}=1-1=0, \quad$ and $Y_{5}=21345678$,

$a_{3}=3-1=2, \quad$ and $Y_{6}=21345678$,

$a_{2}=1-1=0, \quad$ and $Y_{7}=12345678$. The representation of $X$ is 0200353 .

Proposition 4 The representation given above defines a one-one mapping between the $n$-pancake and the $2 \times 3 \times \cdots \times(n-1) \times n$ generalized hypercube. 
Proof. Let $a_{2}, \ldots, a_{p}, p, \ldots, n-1(2 \leq p \leq n-1)$ denote the above representation of a permutation. The proof is by induction on $p$.

- Base: Let $p=2$ and $X=x_{1} x_{2} 34 \cdots(n-1) n$ be a permutation. In that case, $\left\{x_{1}, x_{2}\right\}=\{1,2\}$. If $x_{1}=1$, from rule $(\mathbf{R})$ we have $a_{i}=i-1$ for each value of $i$ (in particular, $a_{2}=1$ ). Similarly, if $x_{1}=2$ we have $a_{2}=0$. Hence, the property holds for $p=2$.

- Induction step: Given $p(2 \leq p \leq n-1)$, suppose the map from the set of permutations $\left\{x_{1} \cdots x_{p}(p+1) \cdots n\right\}$ onto the subgraph of the GHC defined by $\left\{a_{2}, \ldots, a_{p}, p, \ldots, n-1\right\}$ is one-to-one. Let us prove that the property also holds for $(p+1)$.

Consider the permutations $X=x_{1} \cdots x_{p} x_{p+1}(p+2) \cdots n$, and notice that the symbol $(p+1)$ belongs to the set $\left\{x_{1}, \ldots, x_{p} x_{p+1}\right\}$. According to rule $(\mathbf{R})$, the representation of $X$ is constructed step by step, from $i=n$ downto $i=2$, by performing $a_{i}=x_{i}-1$ and exchanging symbols $x_{i}$ and $i$ within $X$. Therefore, $x_{p+1}$ and $(p+1)$ are exchanged in the representation of $X$ and $a_{p+1}$ takes all the values $0,1, \ldots, p$.

Since $X$ is of the form $x_{1} \cdots x_{p}(p+1) \cdots n$, and according to the induction step, the values $a_{i}(1<i<p+1)$ cover the whole set $\{0, \ldots, i-1\}$. Whence the property holds for $(p+1)$.

Now, let $X=a_{2} \cdots a_{i-1} \alpha a_{i+1} \cdots a_{n}$ and $Y=a_{2} \cdots a_{i-1} \beta a_{i+1} \cdots a_{n}$ be the above representations of two permutations. To find the distance from $X$ to $Y$ within the pancake $P_{n}$ we need Lemma 2 first.

Lemma 2 Let two permutations $X$ and $Y$ denoted $a_{2} \cdots a_{i-1} \alpha a_{i+1} \cdots a_{n}$ and $a_{2} \cdots a_{i-1} \beta a_{i+1} \cdots a_{n}$, respectively. Their standard representations differ in at most three positions, i.e. $X=A x B y C z D$ and $Y=A z B x C y D$.

Proof. Consider two permutations $W$ and $Z$, such that $W=A x B p C$, where the symbol $p$ is located at position $p$, with $p>x$, and $Z=A p B x C$. Let us compare each of the respective representations of $W$ and $Z$.

In each representation, the values of $a_{i}$ corresponding to $C$ are equal; the values of $a_{p}$ are $(p-1)$ and $x-1$, and for $i<p, a_{i}$ is obtained from rule $(\mathbf{R})$. The values of the $a_{i}$ s within each representation of $W$ and $Z$ are equal to the symbols in two permutations $\pi_{W}$ and $\pi_{Z}$ (respectively), each obtained by applying rule $(\mathbf{R})$. Now, this construction of $\pi_{W}$ and $\pi_{Z}$ yields $\pi_{W}=\pi_{Z}=\operatorname{ExF} p(p+1) \cdots n$, where $E$ and $F$ are two blocks of symbols in $\{1, \ldots, x\} \backslash\{x, p, \ldots, n\}$. Hence, the representations of $W$ and $Z$ differ in one position only and, for a given $X$, there are $(p-1)$ such $Y$ s. The standard representations of two such $Y$ s differ in three positions, and the proof follows. 
The following Theorem 6 derives from Lemma 2.

Theorem 6 The $2 \times 3 \times \cdots \times(n-1) \times n$ generalized hypercube can be embedded into the n-pancake with dilation 8.

Proof. Let two permutations $X$ and $Y$, whose representations differ in one position only. According to Lemma 2, each of their standard representations differs in at most three positions, i.e. $X=A x B y C z D$ and $Y=A z B x C y D$. A path joining $X$ to $Y$ is thus

$$
\begin{gathered}
X=A x B y C z D \rightarrow z \bar{C} y \bar{B} x \bar{A} D \rightarrow x B y C z \bar{A} D \rightarrow \bar{B} x y C z \bar{A} D \rightarrow \\
B x y C z \bar{A} D \rightarrow \bar{C} y x \bar{B} z \bar{A} D \rightarrow C y x \bar{B} z \bar{A} D \rightarrow y \bar{C} x \bar{B} z \bar{A} D \rightarrow A z B x C y D=Y,
\end{gathered}
$$

and the dilation follows.

Corollary 3 Let $d=1+\lfloor\lg 3\rfloor+\cdots+\lfloor\lg (n-1)\rfloor+\lfloor\lg n\rfloor$ be the dimension of the binary hypercube $Q_{d} . Q_{d}$ can be embedded into the n-pancake with dilation 8.

Proof. $\quad Q_{d}$ is a subgraph of the $2 \times 3 \times \cdots \times(n-1) \times n$ generalized hypercube.

The latter representation of permutations yields an embedding of the GHC into the star graph.

Theorem 7 The $2 \times 3 \times \cdots \times(n-1) \times n$ generalized hypercube can be embedded into the star graph of dimension $n$ with dilation 4.

Proof. Again, the above representation of permutations is used. Let two permutations $X$ and $Y$, wherein at most three symbols have not the same location, i.e. $X=a A x B y C z D$ and $Y=a A z B x C y D$. A path joining $X$ to $Y$ in the $n$-star is thus

$$
\begin{aligned}
X= & a A x B y C z D \rightarrow y A x B a C z D \rightarrow z A x B a C y D \\
& \rightarrow x A z B a C y D \rightarrow a A z B x C y D=Y,
\end{aligned}
$$

and the result follows.

This last theorem improves on the result presented in [8]. Indeed, Nigam et al. show that the binary hypercube can be embedded into the $n$-star with dilation 4. Since the binary hypercube is a subgraph of the GHC, Theorem $\square$ generalizes that result. 


\section{Conclusion}

We presented embeddings of rings, grids, and hypercubes into the pancake interconnection network. All embeddings have constant dilations, and some of them lead to similar results into the star graph. Possible improvements on the above results are twofold.

1. The embedding capabilities offered by the pancake interconnection network are very restrictive. In the present paper, the only embeddings of grids that are considered have size $n \times(n-1)$ ! and $(n+(n-2)+(n-3)+\cdots+(p+1)) \times p !$, for $p=2, \ldots, n-1$. Finding embeddings of $N_{1} \times N_{2}$ grids for all pairs $\left(N_{1}, N_{2}\right)$ such that $N_{1} N_{2} \leq n$ ! would be a much more general result.

2. Some embeddings presented in the paper have congestion $O(n)$. A class of problems of the following kind remains open: find embeddings of the same interconnection networks with constant dilation and congestion, or else, show that such embeddings do not exist.

\section{Acknowledgements}

Many thanks to Houssine Senoussi, who took great part in performing the first version of this work.

\section{References}

[1] S.B. Akers and B. Krishnamurthy. A group theoretic model for symmetric interconnection networks, IEEE Trans. on Computers, Vol. 38, No 4, 1989, 555565 .

[2] M.M. de Azevedo, N. Bagherzadeh and S. Latifi. Variable-Dilation Embeddings of Hypercubes into Star Graphs: Performance Metrics, Mapping Functions, and Routing, Proc. of the 2nd Int. EuroPar Conference, Vol. 1, 1996, 247-252.

[3] L.N. Bhuyan and D.P. Agrawal. Generalized hypercube and hyperbus structures for a computer network, IEEE Trans. on Computers, Vol. 33, 1984, 323-333.

[4] N. Biggs. Algebraic Graph Theory, Cambridge University Press, 1974. 
[5] A. Bouabdallah, M.C. Heydemann, J. Opatrny and D. Sotteau. Embedding complete binary trees into star networks, Proc. of Mathematical Foundations of Computer Science 1994, Lecture Notes in Computer Science 841, 266-275.

[6] J.S. Jwo, S. Lakshmivarahan and S.K. Dhall. Embedding of Cycles and Grids in Star Graphs, Proc. of the 2nd IEEE Symp. on Parallel and Distributed Processing, 1990, 540-547.

[7] Z. Miller, D. Pritikin and I.H. Sudborough. Near Embeddings of Hypercubes into Cayley Graphs on the Symmetric Group, IEEE Trans. on Computers, Vol. 43, No 1, 1994, 13-22.

[8] M. Nigam, S. Sahni and B. Krishnamurthy. Embedding Hamiltonians and Hypercubes in Star interconnection Graphs, Proc. of Int. Conf. on Parallel Processing, 1990, 340-343.

[9] K. Qiu, H. Meijer and S.G. Akl. On the Cycle Structure of Star Graphs, congressus numerantium 96, 1993, 123-141.

[10] K. Qiu, S.G. Akl and H. Meijer. On Some Properties and algorithms for the Star and Pancake Interconnection Networks, J. of Parallel and Distributed Comput. 22, 1994, 16-25. 